\title{
Correction to: Part 1: Slave and Signifier
}

\section{Correction to:}

D. S. Marriott, Lacan Noir, The Palgrave Lacan Series, https://doi. org/10.1007/978-3-030-74978-1_4

Owing to an oversight, the book was published with errors on page 7 . The below changes has been amended.

$S \quad$ changed to $\underline{S}$

$S$ $S$

Figure 1 has been relocated to page 7 from page 21, just under the phrase "With this in mind imagine the following illustration:" (Figure 1) 